\title{
Analysis of the QCD spectrum and chiral symmetry breaking with varying quark masses
}

\author{
Yu.A.Simonov \\ State Research Center \\ Institute of Theoretical and Experimental Physics, \\ Moscow, 117218 Russia
}

December 7, 2018

\begin{abstract}
The meson spectrum of QCD is studied in the framework of nonperturbative QCD as a function of varying quark masses $m_{q}$. It is shown, that the total spectrum consists of two branches: 1) the standard one, which may be called the flux-tube spectrum, depending approximately linearly on $m_{q}$ and 2) the chiral symmetry breaking (CSB) spectrum for pseudoscalar flavor nonsinglet (PS) mesons, with mass dependence $\sqrt{m_{q}}$. The formalism for PS mesons is derived from the QCD Lagrangian with $m_{q}$ corrections, and a unified form of the PS propagator was derived. It is shown, that the CSB branch of PS mesons joins to the flux-tube branch at around $m_{q}=200 \mathrm{MeV}$. All these results are in close correspondence with recent numerical data on large lattices.
\end{abstract}

\section{Introduction}

The connection between the standard QCD picture of confinement, which may be called the flux-tube picture, and the CSB properties, which can be shortly called the chiral physics, is not clearly understood by physical community, especially on the level of model building.

The chiral Lagrangians have been introduced before the QCD era to implement CSB in [1, 2, 3] and the GMOR relation [4] contains an important 
connection between pion characteristics $m_{\pi}, f_{\pi}$ and purely quark characteristics $m_{q}$ and $\langle\bar{q} q\rangle$. From the derivation it is not clear, how this connection will transform with growing $m_{q}$, and in general at what $m_{q}$ chiral mesons cease to be chiral and become standard flux-tube mesons, which do not contain chiral effects, since at large $m_{q}$ CSB is not spontateous any more. On the side of chiral Lagrangians an effective technic of Chiral Perturbation Theory (CPTh) [5, 6] was developed, which allows to calculate all corrections in terms of additional terms, depending on $m_{\pi}, f_{\pi}$. But one of the questions is that correction terms containing $m_{q}$ can occur already in derivation of GMOR, which was actually known only in the chiral limit. This will be demonstrated below explicitly.

To derive GMOR and explicit expressions for $m_{\pi}, f_{\pi}$ etc. we are using the technic of derivation of chiral degrees of freedom in QCD, developed before in [7, 8, 9, 10]. There it is shown explicitly how pionic variables appear in QCD and how they are connected to the quark Green's functions. As a result all chiral dynamics can be expressed in terms of nonchiral (standard fluxtube) quark-aniquark PS Green's function $G^{(0)}(\mathbf{p})$ and the so-called vertex (or residual) mass $M(0)$. Both these quantities are calculated via string tension $\sigma$ and hence one can express chiral dynamics from the first principles.

Another important point is the incorporation of the chiral branch, i.e. $\pi, K, \eta$ and the corresponding radial excited states into the general scheme of mesons, or in other words, to which extent radial excitations of $\pi, K$, e.g. $\pi(2 S), K(2 S)$ etc. are chiral objects.

Finally, studying meson spectra with varying $m_{q}$ one can check the relativistic properties of the Hamiltonian vs explicit numerical data and discover interesting new dependencies. Indeed, as will be shown below, the spectrum of vector mesons depends approximately linearly on $m_{q}$ with good accuracy both in analytic and lattice calculations, while for PS mesons linear dependence for $m_{q}>250 \mathrm{MeV}$ (flux-tube part of spectrum) goes over into the chiral $\sqrt{m_{q}}$ dependence for lower $m_{q}$,

To do all analytic calculations we are using the so-called Relativistic String Hamiltonian (RSH) [11, which contains only first-principle parameters (no constituent masses or subtraction constants) and was used successively for all hadrons: mesons [12], baryons [13], glueballs [14, hybrids [15].

Good agreement in all cases with experiment and lattice data allows to exploit RSH below for a precision test of flux-tube and chiral dynamics in QCD with varying $m_{q}$. 
The plan of the paper is as follows: in section 2 we shortly remind the derivation of GMOR and expressions for $m_{\pi}, f_{\pi}$ in the context of QCD, and find additional terms in $m_{q}$, which are important for growing $m_{q}$. We also compare these results with lattice data.

In section 3 we calculate the flux-tube spectra of vector and PS mesons for the whole set of $m_{q}$ values and compare those in detail with lattice data. We find here linear dependencies of flux-tube spectra.

In section 4 we show how the chiral spectrum is incorporated in the total spectrum and what are chiral corrections to excited pions and kaons. Section 5 contains summary and conclusions.

\section{Quark mass dependence of chiral dynamics}

In the standard approach one considers chiral Lagrangian and chiral perturbation theory as a selfcontained approach and therefore all quark mass dependence (QMD) is usually derived from chiral perturbation theory. In what follows we shall derive chiral dynamics from QCD in a more direct way, as it was done in [7, 8, 9, 10], and in addition we shall calculate correction terms and then define QMD for all basic quantities of chiral dynamics: $m_{\pi}, f_{\pi}$ and $\langle\bar{\psi} \psi\rangle$.

The starting point is the effective quark-meson Lagrangian, derived in [8], where both quark and chiral meson degrees of freedom (d.o.f) are kept,

$$
\begin{gathered}
L_{Q M L}=\int d^{4} x \int d^{4} y\left\{{ } ^ { f } \psi _ { a \alpha } ^ { + } ( x ) \left[\left(i \hat{\partial}+i m_{f}\right)_{\alpha \beta} \delta^{(4)}(x-y) \delta_{f g}+\right.\right. \\
\left.\left.i M \hat{U}_{\alpha \beta}^{(f g)}(x, y)\right]^{g} \psi_{a \beta}(y)-2 n_{f}[J(x, y)]^{-1} M^{2}(x, y)\right\} .
\end{gathered}
$$

Here $\hat{U}_{\alpha \beta}^{(f g)}$ contains Nambu-Goldstone (NG) fields $\phi_{a}, \hat{U}=\exp \left(i \gamma^{5} \phi_{a} t^{a}\right)$, while $M(x, y)$ is the auxiliary scalar field. $J(x, y)$ is expressed as an integral of the confining field correlator $D(x)$, its exact form will not be used below.

After integrating quark d.o.f. one obtains the Effective Chiral Lagrangian $L_{E C L}$, containing effective scalar mass variable $M$ and $N G$ field $\phi_{a}$, with the partition function

$$
Z=\int D M D \phi_{a} \exp L_{E C L}
$$




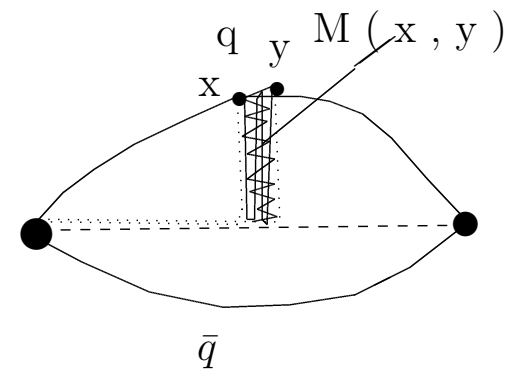

Figure 1: The flux-tube operator $M(x, y)$ in the Wilson loop

where $L_{E C L}$ is

$L_{E C L}=-2 n_{f} \int d^{4} x \int d^{4} y(J(x, y))^{-1} M^{2}(x, y)+N_{c} t r \log \left[\left(i \hat{\partial}+i m_{f}\right) \hat{1}+i M \hat{U}\right]$.

In (11) and (3) $M(x, y)$ enters in its nonlocal form, the nonlocality being of the order of the vacuum correlation length $\lambda \approx 0.1 \mathrm{fm}$. In what follows only the local limit of $M(x, y) \rightarrow M(x)$ will be used. In Fig1. one can see, that $M(x)$ can be associated with the part of the flux tube, from the quark position $x$ to the center of flux tube; the same is true for antiquark.

Expanding the last term in (3) in powers of $\phi_{a}$, one obtains the quadratic vertex in action

$$
W^{(2)}(\phi)=\frac{N_{c}}{2} \int \phi_{a}(k) \phi_{a}(-k) \bar{N}(k) \frac{d^{(4)} k}{(2 \pi)^{4}}
$$

where

$$
\bar{N}(k)=\frac{1}{2} \operatorname{tr}\left\{(\Lambda M)_{0}+\int d^{(4)} z e^{i k z} \Lambda(0, z) M(0) \bar{\Lambda}(z, 0) M(0)\right\} .
$$

The graphical representation of $\bar{N}(k)$ is given in Fig.2.

Here $\phi_{a}(k)$ is

$$
\phi_{a} t_{a}=\frac{\varphi_{a} \lambda_{a}}{f_{\pi}}=\frac{\sqrt{2}}{f_{\pi}}\left(\begin{array}{lll}
\frac{\eta}{\sqrt{6}}+\frac{\pi^{o}}{\sqrt{2}}, & \pi^{+}, & K^{+} \\
\pi^{-}, & \frac{\eta}{\sqrt{6}}-\frac{\pi^{o}}{\sqrt{2}}, & K^{o} \\
K^{-}, & K^{o}, & -\frac{2 \eta}{\sqrt{6}}
\end{array}\right)
$$




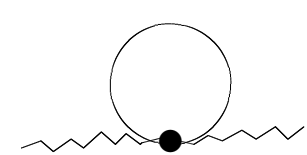

$\mathrm{M}(0)$

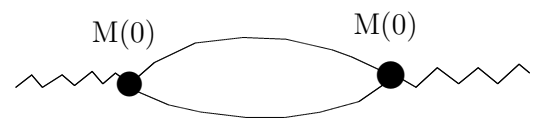

Figure 2: Quadratic terms in the pionic action (5)

and $\Lambda, \bar{\Lambda}$ are defined as

$$
\begin{gathered}
\Lambda=(\hat{\partial}+m+M)^{-1}, \quad \bar{\Lambda}=(\hat{\partial}-m-M)^{-1} \\
\bar{N}(k)=\frac{1}{2} \operatorname{tr}(\Lambda M \bar{\Lambda}(\hat{\partial}-m))=\frac{1}{2}\left[\operatorname{tr}(\Lambda M)_{0}+G^{(M M)}(k)\right]
\end{gathered}
$$

and the last term in (8) is

$$
G^{(M M)}(k)=\operatorname{tr}(\Lambda M \bar{\Lambda} M)_{k}=G^{(M M)}(k)-G^{(M M)}(0)+G^{(M M)}(0) .
$$

Here and in (8) the subscript $(o, k)$ indicates the momentum argument. For $N(0)$ one obtains from (8)

$$
\bar{N}(0)=\frac{1}{2}(\operatorname{tr}(\Lambda m)+\operatorname{tr}(\Lambda m \bar{\Lambda} m)) .
$$

Note the last term on the r.h.s. of (10), which was not calculated before in [7, 8, 9, 10]. Here $m$ is the diagonal quark matrix $m=\operatorname{diag}\left(m_{u}, m_{d}, m_{s}\right)$, and we shall confine ourselves to the $S U(2)$ case, replacing $m$ by $\frac{m_{u}+m_{d}}{2}$.

Now $\operatorname{tr} \Lambda$ can be expressed via the standard chiral condensate, defined in the Minkowskian space-time

$$
\langle\bar{\psi} \psi\rangle_{M}=i\left\langle\psi \psi^{+}\right\rangle_{E}=-N_{c} \operatorname{tr} \Lambda=\langle\bar{u} u+\bar{d} d\rangle_{M}
$$

To trace down the QMD of $\operatorname{tr} \Lambda$ it is convenient to rewrite it as

$$
\operatorname{tr} \Lambda=\left\langle\operatorname{tr}\left(\gamma_{5} \Lambda(x, y) \gamma_{5}(M(0)+m) \Lambda(y, x)\right\rangle d^{4} y=-(M(0)+m) G^{(0)}(k=0) .\right.
$$

In (12) and earlier in (8) we have defined the Green's functions $G^{(0)}$ and $G^{(M M)}$, which differ due to presence in the latter of the operator $M$ at the initial and final points $x$ and $y$. In a general position $M(x)$ contains 
confinement interaction of the given quark with antiquark, i.e. it exemplifies the string between $q$ and $\bar{q}$, see Fig. 1 . However at the initial or the end point of the meson propagator it can be expressed in terms of vacuum correlation length $\lambda[16], \lambda \approx 0.15 \mathrm{fm}$, namely in the Appendix 3 of [9] $M(0)$ was estimated as

$$
M(0)=\frac{2}{\sqrt{\pi}} \sigma \lambda\left(1+O\left(\sigma \lambda^{2}\right)\right) .
$$

As shown in [8, 9, 10] $G^{(0)}$ and $G^{(M M)}$ can be represented as spectral sums over meson states

$$
G^{(0)}(k)=-\sum_{n} \frac{c_{n}^{2}}{k^{2}+m_{n}^{2}}, \quad G^{(M M)}(k)=-M^{2}(0) \sum \frac{c_{n}^{2}}{k^{2}+m_{n}^{2}},
$$

where $c_{n}=\sqrt{\frac{m_{n}}{2}} \psi_{n}(0)$, and $\psi_{n}(\mathbf{x})$ is the $n$-th state meson wave function. As a result one obtains for $\langle\bar{\psi} \psi\rangle$,

$$
|\langle\bar{\psi} \psi\rangle|=N_{c}(M(0)+m) \sum_{n=0}^{\infty} \frac{\left|\psi_{n}(0)\right|^{2}}{m_{n}} .
$$

For $\bar{N}(0)$ from (10), (12) one observes a cancellation of $O\left(m^{2}\right)$ correction terms,

$$
\bar{N}(0)=-\frac{m M(0)}{2} G^{(0)}(0)=\frac{m M(0)}{2} \sum_{n=0}^{\infty} \frac{\left|\psi_{n}(0)\right|^{2}}{2 m_{n}}=\frac{m M(0)}{M(0)+m} \frac{|\langle\bar{\psi} \psi\rangle|}{2 N_{c}}
$$

On the other hand, from the definition of $N(k)$ in (44), one has a correspondence

$$
\bar{N}(k)=\left(m_{\pi}^{2}+k^{2}\right) \frac{f_{\pi}^{2}}{2 N_{c}}+O\left(k^{4}\right)
$$

and as a consequence the (modified) GMOR relation

$$
m_{\pi}^{2} f_{\pi}^{2}=\frac{m M(0)}{M(0)+m}|\langle\bar{\psi} \psi\rangle|,
$$

which goes over into the standard GMOR formula in the chiral limit $m \rightarrow 0$ To find $f_{\pi}^{2}$ separately one can use (8), (9) to write

$$
G^{(M M)}(k)-G^{(M M)}(0)=\frac{k^{2} f_{\pi}^{2}}{2 N_{c}}+O\left(k^{4}\right)
$$


and finally

$$
f_{\pi}^{2}=N_{c} M^{2}(0) \sum_{n=0}^{\infty} \frac{\left|\psi_{n}(0)\right|^{2}}{m_{n}^{3}} .
$$

Eqs. (15), (20) are exploited in the Appendix to obtain numerical values of $\langle\bar{\psi} \psi\rangle$ and $f_{\pi}$ from the calculated spectrum.

Eqs. (15), (18), (20) contain all information about QMD in the corresponding quantities: to make it explicit one needs to tell about QMD of $m_{n}$ and $\psi_{n}(0)$. First of all one should mention, as in [9], that all sums over $n$ in (15), (16), (20) are formally divergent, since spectral representation fails at times and distances less than $\lambda$, and one should use the cut-off factor in integration over small time region, which effectively produces the cut-off factors $e^{-m_{n} \lambda}$ in summation over $n$ in (15) and (20).

This fact, however, does not influence qualitatively the QMD of the corresponding sums over $n$, which is mostly contained in the first terms of these sums, and appears there via $m_{n}$ and $\psi_{n}(0$,

$$
\left|\psi_{n}(0)\right|^{2}=\frac{\omega_{n}}{4 \pi}\left(\sigma+\frac{4}{3} \alpha_{s}\left\langle\frac{1}{r^{2}}\right\rangle\right)
$$

where $\omega_{n}=\left\langle\sqrt{p^{2}+m^{2}}\right\rangle_{n}$.

As will be shown in the next section by analytic calculations and comparison to lattice data, the QMD of $m_{n}, \omega_{n}$ is rather mild, i.e. $m_{n}$ grows by $<20 \%$ when $m_{Q}$ is changing from zero to $200 \mathrm{MeV}$, and in any case this dependence can be computed explicitly. Neglecting it for a moment, one expects with this accuracy:

a) A linear growth of $|\bar{\psi} \psi|$ with $m_{q}$, i.e. for $m_{q} \approx M(0) \approx 0.15 \mathrm{GeV}$ the chiral condensate is twice as large as compared to zero $m_{q}$ value.

b) Approximate independence of $f_{\pi}$ on $m_{q}$ for $m_{q}<0.2 \mathrm{GeV}$ in accordance with Eq. (201).

c) Approximate scaling law $m_{P S} \sim \sqrt{m_{q}}$ for $m_{q} \lesssim 0.2 \mathrm{GeV}$.

More explicitly, $m_{n}$ and $\psi_{n}(0)$ depend on $m_{q}^{2}$, one can expect the following expansions at small $m_{q}$

$$
\begin{aligned}
& m_{P S}^{2}=X m_{q}\left(1+O\left(m_{q}^{2}\right)\right) \\
& f_{P S}\left(m_{q}\right)=f_{P S}(0)+O\left(m_{q}^{2}\right),
\end{aligned}
$$




$$
\langle\bar{\psi} \psi\rangle_{m_{q}}=\langle\bar{\psi} \psi\rangle_{0}\left(\frac{m_{q}+M(0)}{M(0)}\right)\left(1+O\left(m_{q}^{2}\right)\right) .
$$

This should be compared with CPTh results [6]

$$
\begin{gathered}
m_{P S}^{2}=X m_{q}+Y m_{q} \operatorname{lnm}_{q}+Z m_{q}^{2} \\
f_{P S}=f_{0}\left(1+a m_{P S}^{2}\right)+O\left(m_{P S}^{4}\right)
\end{gathered}
$$

Note, that CPTh produces additional terms with respect to our expansions (22)-(24), which originate from higher order terms in expansion of $L_{E C L}$ in powers of $\phi_{a}^{2}$, not accounted in our consideration above.

We can now compare our expansions $(22,-24)$ with lattice data, obtained on large lattices $16^{3} 32$ and $24^{3} 48$ [17]. The detailed comparison in [17] of CPTh expansions (25), (26) with data has shown a good agreement, which supports also our results (22), (23), where no serious deviations from (25), (26) on the basis of proper quark contributions is suggested.

The situation with the chiral condensate in quite different. Indeed, Eqs. (18) and (24) show, that $\langle\bar{\psi} \psi\rangle_{0}$, entering in GMOR relation and $\langle\bar{\psi} \psi\rangle$ at arbitrary value of $m_{q}$, differ by a linear factor $K \equiv \frac{m_{q}+M(0)}{M(0)}$, which can be large, since $M(0) \approx \sigma \lambda \approx O(0.1 \mathrm{GeV})$ (see Appendix 1 of [9]). Additional corrections in $m_{q}$, entering from the sum in (15) are small for small $m_{q}<0.2$ $\mathrm{GeV}$, and the factor $K$ has a clear significance in comparison to lattice data and experiment.

In CPTh the chiral condensate is difficult to treat because of divergence at small $m_{\pi}$. On lattice the condensate $\langle\bar{q} q\rangle$ was studied in numerous papers, see e.g. [18], [19] and [20] for a review and references. Both in [18] and [19] the linear growth of $\langle\bar{\psi} \psi\rangle$ with $m_{q}$ was also observed. As one can see from (15), (20), $f_{\pi} \sim M(0) \sim \sigma \lambda$, while $\langle\bar{q} q\rangle \sim \sigma M(0) \sim \sigma^{2} \lambda$, where we have taken into account, that $\psi_{n}^{2}(0) \sim \omega \sigma \approx \sigma^{3 / 2}, m_{n} \sim \sqrt{\sigma}$. Inserting these estimates into the GMOR relation (18), one obtains

$$
m_{\pi}^{2} \sim m_{q} / \lambda, \quad m_{\pi} \approx \sqrt{m_{q}} \sqrt{M_{G}}
$$

where $M_{G} \approx 1 / \lambda$ is the gluelump mass, obtained in [16], $M_{G} \approx 6 \sqrt{\sigma} \approx 2$ $\mathrm{GeV}$. 


\section{The flux-tube spectrum: $m_{p s}$ and $m_{v}$}

In the previous section the use was made of the spectrum of states $\left\{m_{n}\right\}$ and $\left\{\psi_{n}(0)\right\}$, which correspond to a completely different regime, described by the so-called Relativistic String Hamiltonian, which was derived from the pathintegral representation of the Green's function of quark and antiquark at the ends of the QCD string. Therefore it exemplifies both string asymptotics at large $L$ and relativistic potential dynamics at small $L$. For $L=0$ the RSH for the $q \bar{q}$ system has the form

$$
H=\sum_{i=1}^{2} \sqrt{p^{2}+m_{i}}+V_{\sigma}(r)+V_{g}(r)+\Delta_{S E}+H_{s s},
$$

where $V_{\sigma}(r)=\sigma r, \quad V_{g}(r)=-\frac{4 \alpha_{B}(r)}{3 r}$, and $\Delta_{S E}, H_{s s}$ are self-energy and hyperfine contributions to be defined later.

We shall exploit the so-called einbein version of $\mathrm{RSH}$, which yields results numerically close to (28), but is easier to treat. In this case one introduces the auxiliary variable $\omega_{i}$ and the total mass of the radial excited state $n, n=$ $0,1,2, \ldots$ can be written for equal quark masses $m_{1}=m_{2} \equiv m_{q}, \omega_{1}=\omega_{2}=\omega$, as

$M_{n}(\omega)=\frac{m_{q}^{2}}{\omega}+\omega+\varepsilon_{n}(\omega)+\Delta_{S E}(\omega)+M_{s s}(\omega) \equiv M_{n}^{(0)}(\omega)+\Delta_{S E}(\omega)+M_{s s}(\omega)$

with

$$
\begin{gathered}
\varepsilon_{n}(\omega)=\omega^{-1 / 3} \sigma^{2 / 3} a_{n}(\tau) \quad a_{0}(0)=2.338 ; \quad \tau=\frac{4 \alpha_{s}}{3}\left(\frac{\omega^{2}}{\sigma}\right)^{1 / 3} \\
\Delta_{S E}=-\frac{3 \sigma}{\pi \omega} \eta\left(m_{q} \lambda\right), \quad M_{s s}(\omega)=\frac{8 \alpha_{h f}}{9 \omega^{2}} R_{n}^{2}(0)\left(\mathbf{s}_{1} \mathbf{s}_{2}\right),
\end{gathered}
$$

where $\eta$ is a calculable function, given in [20] note, that $\eta(0)=1$.

In the einbein method the equilibrium point of $M_{n}^{(0)}(\omega)$ is defined by the equation

$$
\left.\frac{d M_{n}^{(0)}(\omega)}{d \omega}\right|_{\omega=\omega_{0}}=0
$$


Table 1: Energy eigenvalues $\varepsilon_{0}\left(m_{q}\right)$ and radial wave function at origin $R_{I S}(0)$ as functions of quark mass

\begin{tabular}{|l|l|l|l|l|l|l|l|l|l|}
\hline \hline$m_{q}(\mathrm{MeV})$ & 0 & 40 & 100 & 200 & 330 & 400 & 500 & 700 & 1400 \\
\hline$\omega_{0}(\mathrm{MeV})$ & 352 & 355 & 373 & 434 & 507 & 578 & 663 & 828 & 1510 \\
\hline$\tau\left(\omega_{0}\right), \alpha_{s}=0.33$ & 0.3885 & 0.391 & 0.409 & 0.447 & 0.495 & 0.541 & 0.5925 & 0.687 & 1.026 \\
\hline$\varepsilon_{0}(\mathrm{MeV})$ & 903 & 900 & 879 & 819 & 760 & 711 & 661 & 583 & 380 \\
\hline$R_{1 S}(0) \mathrm{Gev}^{3 / 2}$ & 0.312 & 0.314 & 0.324 & 0.358 & 0.397 & 0.435 & 0.457 & 0.563 & 0.911 \\
\hline
\end{tabular}

and $\omega_{0}$ acquires the physical meaning of an average quark energy, $\omega_{0}^{(n)}=$ $\left\langle\sqrt{m_{q}^{2}+\mathbf{p}^{2}}\right\rangle_{n}$. Note, that all correction terms, $\Delta_{S E}$ and $M_{s s}$, also depend on $\omega$, and we take them in the first approximation at the point $\omega=\omega_{0}$.

In this way one obtains an equation for $\omega_{0}$,

$$
\omega_{0}^{2}=m_{q}^{2}+\frac{\left(\sigma \omega_{0}\right)^{2 / 3}}{3} a_{n}(\tau)
$$

with solutions which can be written in two forms, the first appropriate for large $m_{q}$,

$$
\omega_{0}^{2}(n)=\sigma\left[\left(\frac{q}{2}+\sqrt{-\frac{p^{3}}{27}+\frac{q^{2}}{4}}\right)^{1 / 3}+\left(\frac{q}{2}-\sqrt{-\frac{p^{3}}{27}+\frac{q^{2}}{4}}\right)^{1 / 3}\right],
$$

while the second is valid for small $m_{q}$, when $\frac{q^{2}}{4}<\frac{p^{3}}{27}$,

$$
\omega_{0}^{2}(n)=\sigma\left[2 \sqrt{\frac{p}{3}} \cos \left(\frac{1}{3} \arctan \left(\frac{2 \sqrt{\frac{p^{3}}{27}-\frac{q^{2}}{4}}}{q}\right)\right)\right]
$$

with $p=\frac{a_{n}}{3}, \quad q=\frac{m_{q}^{2}}{\sigma}$.

The resulting values of $\omega_{0}(n), \varepsilon_{n}\left(\omega_{0}\right)$ are given in Table 1 for $n=0$, $\sigma=0.18 \mathrm{GeV}^{2}$ and different $m_{q}$.

For large $m_{q}, \frac{m_{q}^{2}}{\sigma} \ll 1$, Eq. (32) yields $\omega_{0}^{2} \approx m_{q}^{2}+\frac{\sigma^{2 / 3} a_{n} m^{2 / 3}}{3}$.

One can see in Table 1 , that $\omega_{0}$ change only by $3 \%$, when $m_{q}$ grows from 0 to $100 \mathrm{MeV}$. This fact is basic for small $m_{q}$ corrections to $f_{\pi},\langle\bar{q} q\rangle$ from the sums oven in (15), (20). 
Table 2: Masses of vector state $M_{V}$ and pseudoscalar state $M_{P S}$ together with spin-averaged mass $\bar{M}$ as functions of quark mass

\begin{tabular}{|l|l|l|l|l|l|l|l|l|l|}
\hline \hline$m_{q}(\mathrm{MeV})$ & 0 & 40 & 100 & 200 & 330 & 400 & 500 & 700 & 1400 \\
\hline$M(\mathrm{MeV})$ & 766 & 770 & 874 & 1001 & 1224 & 1357 & 1535 & 1890 & 3154 \\
\hline$M_{V}(\mathrm{MeV})$ & 827 & 830 & 932 & 1053 & 1271 & 1401 & 1572 & 1926 & 3182 \\
\hline$M_{P S}(\mathrm{MeV})$ & 458 & 463 & 578 & 733 & 984 & 1135 & 1349 & 1709 & 3011 \\
\hline
\end{tabular}

We now turn to corrections, $\Delta_{S E}$ and $M_{s s}$, which are defined as in Eq. (30), with $R_{1 S}(0)$ given in the Table 1 , and $\eta\left(m_{q} \lambda\right)$ in the Appendix of [20].

In this way one obtains for the spin-averaged mass $\bar{M}$, at $m_{q}=0$,

$$
\bar{M} \equiv \bar{M}_{\operatorname{cog}}(1 S)=766 \mathrm{MeV},
$$

and for PS and $V$ masses with $\alpha_{h f}=0.35$ and $R_{1 S}(0)$ from Table 1 one would have

$$
M_{V}=827 \mathrm{MeV}, \quad M_{P S}=583 \mathrm{MeV} .
$$

Taking now $V_{s s}$ interaction to the first order into account for the singlet wave function $\psi_{P S}(0)$, one has $\psi_{P S}^{(1)}(0) \simeq 1.3 \Psi_{P S}^{(0)}(0)$, which shifts $M_{P S}$ down, $M_{P S}^{(!)}=458 \mathrm{MeV}$.

We keep this procedure for all $m_{q}$ and get in this way the values of $M_{P S}$ and $M_{v}$, given in Table 2 .

To make comparison with lattice data we demonstrate in Table 3 numerical values of $M_{P S}, M_{V}$ and $f_{P S}$, obtained in [17] on large lattices $16^{3} 32$ and $24^{3} 48$ at $\beta=8.00$ and 8.45 respectively. The corresponding values of $m_{q}$ were found using the standard procedure with chiral behavior of $M_{P S}\left(m_{q}\right) \sim \sqrt{m_{q}}$, as it is also clear from the last column of Table 3. Comparison of the values of $M_{V}\left(m_{q}\right)$ in Table 3 and Table 2 shows an agreement within the accuracy of $10 \% 1$. The same can be told about the $M_{P S}$ values for $m_{q}>m_{\text {crit }} \approx 200$ $\mathrm{MeV}$, however for smaller $m_{q}$ the RSH calculation predicts $M_{P S}$ slowly changing with a finite value $O(400 \mathrm{MeV})$ for $m_{q}=0$. This implies that chiral regime is outside of $\mathrm{RSH}$ and around $m_{q}=200 \mathrm{MeV}$ a change of regimes takes place.

\footnotetext{
${ }^{1} \mathrm{~A}$ few percent agreement occurs for $\alpha_{s} \approx 0.4$, but for illustrative purposes we keep in Tables 1 and $2 \alpha_{s}=0.33$.
} 
Table 3: Masses $M_{P S}, M_{V}$ and $f_{P S}$ (all in $\mathrm{MeV}$ ), calculated in [17], as functions of quark mass.

\begin{tabular}{|l|l|l|l|l|}
\hline \multicolumn{5}{|c|}{$16^{3} 32, \quad \beta=8.00$} \\
\hline$m_{q}(\mathrm{MeV})$ & $M_{P S}$ & $M_{V}$ & $f_{P S}$ & $\frac{m_{P S}}{\sqrt{m_{q}} \mathrm{Gev}^{1 / 2}}$ \\
\hline 13.35 & 239 & 809 & 94.3 & 2.068 \\
\hline 35.2 & 295 & 816 & 96.1 & 1.57 \\
\hline 52.8 & 353 & 829 & 98.1 & 1.53 \\
\hline 70.4 & 403 & 841 & 100.6 & 1.518 \\
\hline 105.6 & 488 & 874 & 103.1 & 1.50 \\
\hline 176 & 631 & 944 & 113.27 & 1.50 \\
\hline 246.5 & 753 & 1025 & 122 & 1.516 \\
\hline \multicolumn{5}{|c|}{$24^{3} 48, \beta=8.45$} \\
\hline 300 & 887 & 1286 & 153 & 1.62 \\
\hline 211 & 731 & 1159 & 140 & 1.59 \\
\hline 120 & 551 & 1036 & 125 & 1.59 \\
\hline
\end{tabular}

One can see in Fig. 3 that our vector masses $M_{V}$ as a function of $m_{q}$ with good accuracy lie on the sequence of two straight lines, and the RSH values of PS masses are on an almost parallel line for $m_{q}>m_{\text {crit }}$, while the chiral branch for $m_{q}<m_{\text {crit }}$ follow the law $M_{P S}^{2} \sim m_{q}$, which is in agreement with lattice data, shown on Fig.3.

In the next section we shall discuss this change of regimes from another side of the chiral approach and a possibility of incorporating two regimes into one scheme.

It is interesting, that the entries in the rightest column of Table 3, are according to (27), the square root of the gluelump mass, $\sqrt{M_{G}}$, which is approximately equal in Table 3 to $1.5 \mathrm{GeV}^{1 / 2}$, and $M_{G} \approx O(2 \mathrm{GeV})$, as was predicted in (27),

\section{A universal spectrum in the PS channel}

The corrected GMOR relation (18) gives the values of the PS masses in the wide interval for $m_{q}<m_{\text {crit }}$, where the square root behavior $M_{P S} \sim \sqrt{m_{q}}$ goes over into the quasilinear RSH regime $M_{P S} \sim a+b m_{q}$. One might won- 
der how the quasilinear regime,present in the spectrum of the RSH Green's function $G^{(0)}(k)=\sum_{n} \frac{c_{n}^{2}}{k^{2}+m_{n}^{2}}$ is coexisting with the chiral regime in the "total" Green's function $G(k)$. To this end one can compare definitions of $N(k)$ in (44), (5) and (17) and understand, that $\frac{2 N_{c}}{f_{\pi}^{2}} N(k)=m_{\pi}^{2}+k^{2}+O\left(k^{4}\right)$ is the inverse of the generalized pion propagator,

$$
\Delta_{\pi}(k) \equiv \frac{f_{\pi}^{2}}{2 N_{c} N(k)}=\frac{1}{m_{\pi}^{2}+k^{2} a\left(k^{2}\right)}
$$

where

$$
a\left(k^{2}\right)=\frac{2 N_{c} M^{2}(0)}{f_{\pi}^{2}} \sum_{n=0}^{\infty} \frac{c_{n}^{2}}{m_{n}^{2}\left(k^{2}+m_{n}^{2}\right)} \equiv \frac{f_{\pi}^{2}\left(k^{2}\right)}{f_{\pi}^{2}(0)}
$$

Note that $a(0)=1$ due to (20) .

From (36) one can find poles of $\Delta_{\pi}(k)$, which are connected to the poles in $a\left(k^{2}\right)$. The latter are standard flux-tube eigenstates, the spin-averaged ones, since $G(k)$ does not contain hyperfine interaction. As a consequence one obtains for $m_{q}=0$

$$
a\left(k^{2}\right) \text { : poles at } \quad-k^{2}=m_{n}^{2}, \quad n=0,1,2, \ldots
$$

$$
\Delta_{\pi}(k) \text { : poles at } k^{2}=0, ;-k^{2}=m_{n}^{2}-\delta m_{n}^{2} \text { (chiral), } n=1,2, \ldots
$$

Note, that the $m_{0}^{2}$ pole is replaced in $\Delta_{\pi}(k)$ by the pion pole (in the chiral limit) $k^{2}=0$, while $m_{1}^{2}$ is shifted down by $\delta m_{1}^{2}$ (chiral).

One can check, that for the initial values $m_{0} \simeq 0.5 \mathrm{GeV}, m_{1}=1.3 \mathrm{GeV}$, the shift of the mass eigenvalue of $m_{\pi}(2 S)$ is around $0.15 \mathrm{GeV}$. This means, that the lowest $1 \mathrm{~S}$ eigenvalue of the RSH at $m_{0}$ is replaced by $m_{\pi}$, and the $2 \mathrm{~S}$ state is shifted down by $\sim 10 \%$, which is expected from the physical considerations, since this shift replaces the hyperfine interaction.

In the general case of $m_{q}>0$ the poles of $\Delta_{\pi}(k)$ are at $k^{2}=-M_{\text {unif }}^{2}$ where $M_{\text {unif }}^{2} \approx \frac{m_{\pi}^{2}\left(m_{q}\right) m_{0}^{2}\left(m_{q}\right)}{m_{\pi}^{2}\left(m_{q}\right)+m_{0}^{2}\left(m_{q}\right)}\left(1+O\left(\frac{m_{\pi}^{2}\left(m_{q}\right)}{m_{1}^{2}\left(m_{q}\right)}\right)\right)$.

The situation with poles is illustrated in Fig. 4, where poles of $\Delta_{\pi}(k)$ denoted as $M_{\text {unif }}$ are shown together with lattice values of $M_{\text {Lat }}$ and the fluxtube values of $M_{P S}$, taken from Table 2 . One can see, that $M_{\text {unif }}$ approaches $M_{P S}$ with growing $m_{q}$ and all three branches $M_{P S}, M_{\text {Lat }}$ and $M_{\text {unif }}$ are rather close to each other for $m_{q}>150 \mathrm{MeV}$. Thus indeed, unification of chiral and flux-tube dynamics, automatically obtained on the lattice can be paralleled with our explicit mechanism of unification, given in (36). 


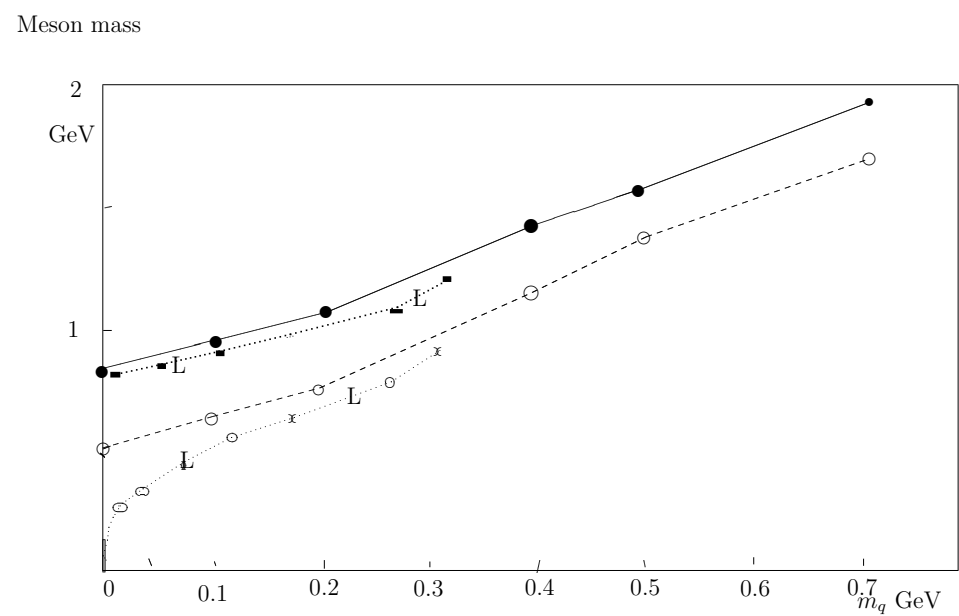

Figure 3: Spectrum of vector and PS mesons from analytic calculations with the Hamiltonian (28) (solid and dashed lines respectively) and from lattice calculations [17] (dotted lines marked by the letter $L$ ). 


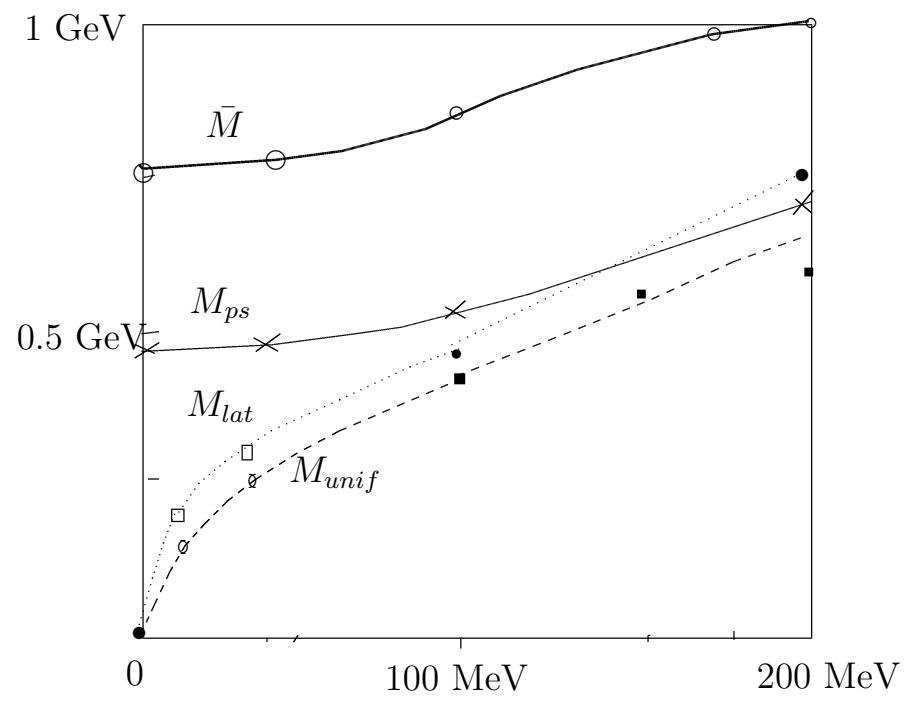

Figure 4: Masses of PS mesons as functions of quark mass; $M_{P S}$ calculated in the flux-tube dynamics (solid line), $M_{\text {lat }}$, calculated on the lattice from [17], and $M_{\text {unif }}$, as poles of the generalized pion propagator, Eq.(36). The upper solid line is for the spin-averaged masses $\bar{M}$, from Table 2 . 


\section{Summary and conclusions}

We have studied in the paper above the confining and CSB dynamics using varying quark masses as a tool to distinguish in the hadron spectra different branches, corresponding to different mechanisms. Comparison to the existing lattice data allows to establish a good accuracy of our relativistic formalism, based on RSH, and an interesting correspondence between the CSB dynamics and the flux-tube dynamics, and to answer the question, at what quark mass the Nambu-Goldstone modes transform into the standard flux-tube modes. In section 3 we have found, that it happens approximately at $m_{q}=m_{\text {crit }} \approx$ $0.2 \mathrm{GeV}$. Another interesting point is that the almost linear dependence of $m_{V}$ on $m_{q}$ slightly changes its slope at $m_{\text {crit }}^{\prime}=\sqrt{2 \sigma}\left(\frac{a_{n}}{9}\right)^{3 / 4} \approx 0.21 \mathrm{GeV}$ (cf. Eqs. (33) and (34)), which again agrees with lattice and analytic calculations, as can be seen from Fig. 3 .

Maybe the most important result of the paper is the improved derivation of the chiral dynamics directly from the QCD Lagrangian in section 2, which enables one not only to obtain GMOR relation, but also find also the corresponding $m_{q}$ dependencies. This program, started in [7, 8, 9, 10], allows in principle to derive the chiral Lagrangian directly from QCD, including higher-order corrections, and as was already mentioned above, to connect chiral and flux-tube dynamics, which are usually treated separately.

Note the important role, which is played in this program by the new entity - the vertex (or residual) mass $M(0)$. Its value was calculated in terms of $\sigma$ and the correlation length $\lambda, M(0) \sim \sigma \lambda$. Note, that in the flux-tube dynamics, i.e. at distances $\langle r\rangle \approx 1 / \sqrt{\sigma}$, the mass $M(0)$ does not appear, and it is needed only to calculate $f_{\pi}$ and $\langle\bar{q} q\rangle$, while in the GMOR relations $M(0)$ is absent.

Looking from the flux-tube dynamics side, it is interesting to try to understand, which kind of forces causes the breakdown of flux-tube and appearance of chiral dynamics, and in particular the strong reduction of the pion mass. To this end consider in particular, the hyperfine $(h f)$ interaction, deduced in the RSH, which is singular for small values of $\omega_{i}$ (i.e. for small average quark energies), since

$$
M_{s s}(\omega)=\frac{8}{9} \frac{\alpha_{h f}}{\omega} \sigma\left(\mathbf{s}_{i} \mathbf{s}_{j}\right)
$$

Taking this into account and working in the einbein formalism, one can 
find minimum of $M\left(\omega_{i}=\omega_{j}\right)$ in the PS channel,

$$
M(\omega)=\frac{m_{q}^{2}}{\omega}-\frac{2}{3} \frac{\alpha_{h f} \sigma}{\omega}+O\left(\omega^{-1 / 3}\right) .
$$

This minimum exists for $m_{q}>m_{\text {crit }}^{\prime \prime}=\sqrt{\frac{2}{3} \alpha_{h f} \sigma} \approx 0.2 \mathrm{GeV}$, but disappears for smaller $m_{q}$, and $M(\omega)$ is not bounded from below.

Usually and in the present paper the $h f$ interaction is treated perturbatively, which in particular means, that the corresponding $\omega$ in $M_{h f}$ is taken from the spin-averaged masses $M^{(0)}$.

It is not known at present how to treat $M_{h f}$ nonperturbatively for $m_{q}<$ $m_{c r i t}^{\prime \prime}$. It seems very likely, that the divergence of $M$ for small $\omega$ implies necessity of consideration of multiple pair creation or, in other words, the reconstruction of the vacuum with the appearance of quark pair condensate. This is still another manifestation of the appearance of the new regime - the chiral Nambu-Goldstone regime.

From this point of view it is probably not surprising, that all three critical masses $m_{\text {crit }}, m_{c r i t}^{\prime}, m_{c r i t}^{\prime \prime}$ approximately coincide. This fact calls for further studies with the aim of understanding and unifying chiral and flux-tube dynamics.

The author is grateful to A.M.Badalian for constant help and useful advices.

\section{Appendix 1}

\section{Calculation of $f_{\pi}$ and $\langle\bar{q} q\rangle$}

We illustrate in the appendix the methods of section 2 with an improved calculation of the chiral condensate and $f_{\pi},\langle\bar{q} q\rangle$ using Eq.(15) and (20) respectively and the methods, given in [8, 9, 10].

Defining these values at $\lambda^{-1}=2 \mathrm{GeV}$, one should use the corresponding cut-off factors, and one has 2

\footnotetext{
${ }^{2}$ Extra factors in (A1.1), (A1.2), as compared to (15), are due to exclusion of a piece of the Euclidean time integration from 0 to $\lambda$, see eq. (11) of [9].
} 


$$
\begin{aligned}
& -\frac{\langle\bar{q} q\rangle}{n_{f}}=N_{c}\left(M(0)+\bar{m}_{q}\right) \sum_{n=0}^{N} \frac{\psi_{n}^{2}(0)}{m_{n}} e^{-m_{n} \lambda} \\
& f_{\pi}^{2}=N_{c} M^{2}(0) \sum_{n=0}^{N} \frac{\psi_{n}^{2}(0)}{m_{n}^{3}} e^{-m_{n} \lambda}\left(1+m_{n} \lambda\right) .
\end{aligned}
$$

Here $N$ is taken to be 2 , since higher terms are small.

Insertion of $m_{n}, \psi_{n}^{2}(0)$ for $n=0,1,2$ from the $\mathrm{RSH}$ in section 3 , one obtains

$$
\begin{gathered}
-\frac{\langle\bar{q} q\rangle^{(2 \mathrm{GeV})}}{n_{f}}=(217 \mathrm{MeV})^{3} \frac{\left(M(0)+m_{q}\right)}{150 \mathrm{MeV}} \\
f_{\pi}^{(2 \mathrm{GeV})}=96 \mathrm{MeV} \frac{\left(M(0)+m_{q}\right)}{150 \mathrm{MeV}} .
\end{gathered}
$$

This should be compared with the values, obtained on the lattice [17, 18, 19, 20], which can be roughly characterized by an average value in the quenched case $-\langle\bar{q} q\rangle=[(270 \pm 20) \mathrm{MeV}]^{3}$ while from [20] for $n_{f}=2$

$$
-\frac{\langle\bar{q} q\rangle}{n_{f}}=(209 \pm 8 \mathrm{MeV})^{3} .
$$

The results (A1.1), (A1.2) are sensitive to the cut-off factor (vacuum correlation length) $\lambda$, e.g. for $\lambda=1.5 \mathrm{GeV}^{-1}$ one obtains

$$
\begin{gathered}
-\frac{\langle\bar{q} q\rangle^{1.5 \mathrm{GeV}}}{n_{f}}=(195 \mathrm{MeV})^{3} \frac{\left(M(0)+m_{q}\right)}{120 \mathrm{MeV}} \\
f_{\pi}^{1.5 \mathrm{GeV}}=64 \mathrm{MeV}\left(\frac{\left(M(0)+m_{q}\right)}{120 \mathrm{MeV}}\right) .
\end{gathered}
$$

In the paper we adopt the results (A1.3), (A1.4) and the corresponding estimate $M(0)=0.15 \mathrm{GeV}$ as our current values.

\section{References}

[1] S.L.Glasow and S.Weinberg, Phys. Rev. Lett. 20, 224 (1968).

[2] S.Weinberg, Physica, 96 A, 327 (1979). 
[3] M.Gell-Mann and M.Lévy, Nuovo Cim. 1653 (1960).

[4] M.Gell-Mann, R.L.Oakes and B.Renner, Phys. Rev. 175, 2195 (1968).

[5] J.Gasser and H.Leutwyler, Phys. Rep. C 87, 77 (1982); Ann. Phys. (N.Y.) 158, 142; Nucl. Phys. B250, 465 (1985).

[6] J.Gasser and H.Leutwyler, Nucl. Phys. B250, 465 (1985); C.W.Bernard and M.F.L.Golterman, Phys. Rev. D 46, 853 (1992); S.R.Sharpe, Phys. Rev. D 46, 3146 (1992).

[7] Yu.A.Simonov, Phys. Rev. D 65, 094018 (2002); hep-ph/0201170.

[8] Yu.A.Simonov, Phys. At. Nucl. 67, 846 (2004); hep-ph/0302090.

[9] Yu.A.Simonov, Phys. At. Nucl. 67, 1027 (2004); hep-ph/0305281.

[10] S.M.Fedorov and Yu.A.Simonov, JETP Lett. 78, 57 (2003); hep-ph/0306216.

[11] A.Yu.Dubin, A.B.Kaidalov and Yu.A.Simonov, Phys. Atom. Nucl. 56, 1745 (1993); Phys. Lett. B 323, 41 (1994); Yu.S.Kalashnikova, A.V.Nefediev and Yu.A.Simonov, Phys. Rev. D 64, 014037 (2001).

[12] A.M.Badalian and B.L.G.Bakker, Phys. Rev. D 66,034025 (2002); A.M.Badalian, B.L.G.Bakker, Yu.A.Simonov, Phys. Rev. D 66, 034026 (2002).

[13] Yu.A.Simonov, Phys. At. Nucl. 66, 338 (2003); Phys, Rev. D 65, 116004 (2002).

[14] A.B.Kaidalov and Yu.A.Simonov, Phys. Lett. B 477, 163 (2000), ibid. B 636, 101 (2006).

[15] Yu.S.Kalashnikova, and D.S.Kuzmenko, Phys. At. Nucl. 67, 538 (2004), ibid 66, 955 (2003).

[16] Yu.A.Simonov, Trudy Math. Inst. V.I.Steclova, V. 272, p. 234 (2001); arXiv: 1003.3608; Yu.A.Simonov and V.I.Shevchenko, Adv. High Energy Phys., 2009:873051 (2009); arXiv: 0902.1405.

[17] D.Galletly, M.Gürtler, R.Horsley, H.Perlt et al., (QCD SF- UK QCD Collaboration), hep-lat/0607024. 
[18] T.B.Blum, P.Chen, N.Christ et al., Phys. Rev. D 69, 074502 (2004).

[19] V.Gimenez, V.Lubicz, F.Mescia, V.Porretti and J.Reyes, Eur. Phys. J. C 41, 535 (2005); hep-lat/0503001.

[20] C.McNeile, hep-lat/0504006; G.Colangelo, Nucl. Phys. Proc. Suppl. 140, 120 (2005); M.R.Pennington, hep-ph/0207220.

[21] Yu.A.Simonov, Phys. Lett. B515, 137 (2001); hep-ph/0105141;

A.Di Giacomo and Yu.A.Simonov, Phys. Lett. B595, 368 (2004). 